\title{
Modern techniques to optimize neuraxial labor analgesia
}

Received June 18, 2018

Revised June 21, 2018

Accepted June 29, 2018

\section{Jalal A. Nanji and Brendan Carvalho}

Department of Anesthesiology, Perioperative and Pain Medicine, Stanford University School of Medicine, Stanford, CA, USA

\author{
Corresponding author \\ Brendan Carvalho, MBBCh, FRCA, \\ $\mathrm{MDCH}$ \\ Department of Anesthesiology, \\ Perioperative and Pain Medicine, \\ Stanford University School of \\ Medicine, 300 Pasteur Drive, MC: \\ 5640, Stanford, CA 94305, USA \\ Tel: 1-650-724-2614 \\ Fax: 1-650-725-8544 \\ E-mail: carvalb@stanford.edu \\ ORCID \\ http://orcid.org/0000-0002-4919-4542
}

Neuraxial analgesia is the gold standard method for pain relief in labor. Several techniques can optimize both the initiation and maintenance of neuraxial labor analgesia. Initiation techniques such as combined spinal-epidural or dural puncture epidural may offer some advantages over standard epidural insertion. The use of ultrasound to assist with landmarking and optimizing block placement improves neuraxial success, and is particularly useful in certain patient populations. Maintaining labor analgesia with a regimen that includes background programmed intermittent boluses with a patientcontrolled epidural analgesia component affords the best combination of pain relief and avoidance of undesired effects. These techniques are most effective when dilute local anesthetics with lipophilic opioids are utilized.

Key Words: Epidural analgesia, Neuraxial labor analgesia, Obstetrical analgesia, Pain management, Patient-controlled analgesia.

\section{INTRODUCTION}

The pain of labor ranks consistently among the most severe types of pain that a woman will experience during her lifetime. There are many pharmacologic and non-pharmacologic options for pain relief during labor, but neuraxial analgesia remains the most effective and is the gold standard against which other modalities are compared. Neuraxial techniques, for initiation and maintenance, have evolved significantly over the past several decades, and modern techniques allow labor neuraxial analgesia to be administered extremely effectively and safely for both mother and unborn child. Consequently, neuraxial analgesia is an increasingly requested and utilized modality for the provision of labor analgesia [1]. This review will provide a brief overview of key, state-of-the-art techniques for the initiation and maintenance of neuraxial analgesia for labor pain relief.

\section{TECHNIQUES TO OPTIMIZE NEURAXIAL LABOR ANALGESIA INITIATION}

\section{Combined spinal-epidural (CSE)}

Numerous studies and meta-analysis have demonstrated that CSE results in significantly quicker onset of labor analgesia compared to a standard epidural [2]. In addition, it may result in superior pain relief with reduced requirement for supplemental dosing [3], fewer unilateral blocks [4], fewer epidural catheter failures [5], and improved maternal satisfaction [6].

Booth et al. [5] found a 7\% failure rate with CSE compared to $12 \%$ with standard epidural. The authors demonstrated that epidural catheter failures were also recognized early (within 30 minutes) more frequently with CSE compared to standard epidural. This lower failure rate with CSE may be due to fewer false losses-of-resistance, as visualizing cerebrospinal fluid (CSF) in the hub of the spinal needle confirms

This is an Open Access article distributed under the terms of the Creative Commons Attribution Non-Commercial License (http://creativecommons.org/licenses/by-nc/4.0) which permits unrestricted non-commercial use, distribution, and reproduction in any medium, provided the original work is properly cited. 
correct epidural placement of the Tuohy needle. In support of this argument, Grondin et al. [7] found that in the absence of CSF return during the spinal portion of the CSE, $29 \%$ of epidural catheters required subsequent replacement, compared to only $4 \%$ of catheters placed after initial CSF return was confirmed. The observation of CSF with the CSE technique also confirms midline placement, and may explain the lower incidence of unilateral blocks with CSE compared to standard epidurals [4]. Epidural catheters placed as part of a CSE technique appear less likely to fail than epidurals (odds ratio $[\mathrm{OR}]=5.5$ for failure of epidural compared to CSE) when attempting conversion from labor analgesia to anesthesia for cesarean delivery $(\mathrm{CD})[8]$.

The CSE technique does, however, increase the incidence of fetal bradycardia and heart rate abnormalities immediately following block placement compared to epidural alone (relative risk $[R R]=1.3$ ) [9]. The proposed mechanism for fetal bradycardia is a sudden decrease in circulating epinephrine after rapid onset of analgesia with CSE, resulting in uterine hypertonus and transiently diminished uteroplacental flow $[10,11]$. The risk of fetal bradycardia is increased in advanced labor with high pain levels [12], and may also increase with higher doses of intrathecal opioids [13]. Fetal bradycardia following CSE is usually easily managed with tocolytics and maternal blood pressure management, and does not appear to impact $\mathrm{CD}$ rates or neonatal outcomes $[2,9]$.

CSE may be associated with increased maternal side effects including hypotension and opioid-induced pruritis [2]. The CSE technique may result in a lower incidence of unintended dural puncture compared to standard epidural, but no differences in need for epidural blood patch were found in a large, retrospective review of over 19,000 patients [14]. The theoretical concern that the epidural catheter placed as part of a CSE technique remains "untested" until the spinal component has worn off is likely unfounded [15], as CSE is associated with lower failure rates for labor or CD anesthesia compared to standard epidural $[5,8]$.

\section{Dural-puncture Epidural (DPE)}

A DPE is a more recently described epidural initiation technique intended to reduce some of the side effects (e.g., fetal bradycardia, pruritus) of CSE, while improving on the standard labor epidural. The DPE technique is similar to CSE in that a small-gauge spinal needle is inserted through the Touhy epidural needle after achieving loss of resistance (LOR) ("needle-thru-needle" technique), however unlike CSE no intrathecal medications are administered after the dura is punctured. The postulated benefit of DPE over standard epidural is that the intentional dural puncture with the spinal needle provides a conduit for epidurally-administered medications to translocate into the subarachnoid space. In addition, positive return of CSF serves to confirm that the epidural needle is in the epidural space in a midline trajectory.

Studies have demonstrated marginally quicker onset of analgesia, better sacral coverage, and decreased incidence of unilateral block with DPE compared to epidural [16]. A recent study by Chau et al. [17] compared the DPE technique with both CSE and standard epidural for labor analgesia. They found that the CSE technique provided significantly quicker (2 [1-6] minutes) compared to both the DPE (11 [4-120] minutes) and standard epidural (18 [10-120] minutes) (reported as median [interquartile range] time to numeric pain rating scale $<1)$. However, there was a reduced need for subsequent anesthesia provider interventions in the DPE group compared to both the standard epidural and CSE techniques. DPE was also associated with fewer side effects including pruritis, maternal hypotension, and uterine hypertonus compared to the CSE.

The efficacy of the DPE technique likely depends on the use of a $25-\mathrm{G}$ or larger spinal needle. One study found that the magnitude of local anesthetic flux across the dura is dependent on the size of the dural puncture, and was significantly increased only by puncture with a 24-G Sprotte needle compared to a 27-G Whitacre needle [18]. Thomas et al. [19] found that puncturing the dura with a 27-G needle as part of a DPE did not improve analgesia when compared to a standard epidural. The post-dural puncture headache risk with DPE using 24-25 G needles has not been well-elucidated.

The optimal role for DPE as a labor analgesic technique has therefore not been fully resolved. A CSE remains the preferred technique if quick-onset analgesia in advanced labor is desired. The DPE technique may be a useful technique to confirm LOR or midline insertion when equivocal LOR is obtained, or after difficult block placement when anatomical landmarks make midline assessment difficult. Confirmation of CSF flow through the spinal needle inserted through the epidural needle after LOR provides reassurance that the epi- 
dural space was indeed encountered, and midline obtained.

\section{Ultrasound-assisted neuraxial techniques}

Along with the increasing use of ultrasound (US) in anesthesia practice for applications such as regional nerve blockade, vascular access, and point-of-care ultrasound, there has been a recognition of the utility of US in aiding with neuraxial block insertion [20]. The role of US to assist neuraxial insertion has primarily been limited to pre-procedure landmarking and depth assessment, as real-time US-guided neuraxial anesthesia remains technically difficult for a single operator. A pre-procedure US allows for the following information to be determined prior to starting the epidural procedure: accurate interspace identification, establishment of midline, estimation of depth to epidural space, as well as the determination of the optimal interspace, insertion point and Tuohy needle angulation.

The accuracy of the estimated depth at which LOR will actually occur with a Tuohy needle as measured by US is very high (correlation coefficient $=0.91$ ) [21]. When compared to traditional landmark technique, US-assisted lumbar neuraxial procedures are also associated with decreased rates of both technical failure $(R R=0.51)$ and traumatic insertion $(R R=$ 0.27 ) [21], as well as fewer needle insertions and redirections [22]. Neuraxial US is also an extremely valuable teaching tool in academic institutions, with its use resulting in improved success of trainees when first learning neuraxial placement and a reduction in the number of subsequent catheter replacements $[23,24]$. The advantages of US-assistance, however, may not be applicable to more experienced providers or in patients with easily palpable landmarks [25].

Some studies have described superior labor analgesia and higher maternal satisfaction associated with US use, perhaps due to increased frequency of midline placement and optimal interspace selection [26]. A lower (L4/5) interspace provides better perineal pain relief due to better sacral coverage during the second stage of labor compared to a higher (L1/2) interspace epidural insertion [27]. Anesthesia providers are able to correctly identify the interspace using the traditional landmarks of iliac crests and intercristal (Tuffier's) line $29 \%-41 \%$ of the time $[21,28]$. The use of US may result in placement of neuraxial block in lower interspaces that are more likely to result in adequate analgesia during the second stage of labor.

Suggested clinical indications for the use of lumbar neuraxial US include anticipated difficulty with block placement (e.g., obesity, scoliosis, history of failed or difficult neuraxial block) or to assist with block placement when the traditional landmark-based approach has failed or is technically challenging. Utilizing US to assist with neuraxial placement can be particularly useful in women with scoliosis, previous spinal surgery, obesity [29], or impalpable bony landmarks [30].

\section{TECHNIQUES TO OPTIMIZE NEURAXIAL ANALGESIA MAINTENANCE}

\section{Patient-controlled epidural analgesia (PCEA)}

Techniques to optimize epidural labor analgesia have evolved significantly over the past several decades. When epidurals were first utilized for labor pain management, the primary method of maintaining analgesia was provideradministered boluses by anesthesiologists, midwives or obstetricians. These intermittent boluses, given in response to patient discomfort or at standard time intervals, were effective but labor-intensive. With the introduction of automated epidural pumps, continuous epidural infusions (CEI) became the preferred technique to maintain epidural labor analgesia. PCEA for labor was first described by Gambling et al. [31] in 1988. Compared to CEI, PCEA has been shown to decrease local anesthetic requirements, reduce motor block, decrease the need for provider-administered top-ups, and in some studies improve analgesia and maternal satisfaction [32]. The majority of patients in the United States are now offered PCEA for epidural labor analgesia maintenance [33]. Better pain relief and reduced provider call backs for additional top-ups can be achieved when a background CEI is added to PCEA compared to PCEA alone [34], although at the expense of increased overall local anesthetic consumption.

\section{Programmed intermittent epidural boluses (PIEB)}

In 2004, PIEB or automated mandatory boluses was proposed as a more optimal neuraxial labor analgesia maintenance technique compared to CEI [35]. With PIEB, an epidural pump provides automated boluses of epidural medications administered at set intervals (e.g., every 45 minutes). The 
theory that underpins the efficacy of PIEB compared to CEI is that spread of medications is not uniform in the epidural space [36] and that boluses given rapidly through an epidural catheter spread better than when the same hourly volume is given by slow infusion [37]. This increased spinal segmental spread has been well-demonstrated in a porcine model [38].

Wong et al. [39] demonstrated prospectively that PIEB + PCEA resulted in lower local anesthetic requirements, improved maternal satisfaction, and fewer manual rescue boluses compared to CEI + PCEA, and Capogna et al. [40] found that PIEB + PCEA compared to CEI + PCEA reduced maternal motor block and instrumental vaginal delivery. Two systematic reviews and meta-analyses $[41,42]$ have demonstrated the following advantages of PIEB when compared to CEI (with or without PCEA): decreased local anesthetic usage, improved maternal satisfaction, and less breakthrough pain. Impact studies after the introduction of PIEB + PCEA to replace CEI + PCEA at various institutions have shown modest improvements in outcomes including reduced need for clinician boluses, less frequency of unilateral block, and decreased peak pain scores [43-45].

In summary, the data that the optimal method of maintenance of epidural labor analgesia is PCEA with a background PIEB. PIEB allows for provision of a baseline level of analgesia, and PCEA allows for further individualization of dosing to account for variability in progress of labor and differences in patient preference.

\section{Optimal PCEA and PIEB settings}

Numerous studies have tried to determine the optimal settings (or recipe) for PIEB + PCEA for labor analgesia maintenance. Table 1 shows an example of such a recipe used at Stanford University in the United States. The epidural solution (local anesthetic and opioid concentration), programmed/automated and demand bolus volume, the PIEB time interval, and lockout intervals for both PIEB and PCEA can all impact the efficacy of the technique. A systematic review and a more recent implementation article $[34,46]$ highlight the various recipes that have been used for both PCEA and PIEB for labor analgesia. Although there is no one optimal PCEA setting for epidural labor analgesia, studies suggest a larger bolus given less frequently is more preferable, although excessive lockout intervals may increase provider call-backs [34]. Wong et al. [39] found that a PIEB interval of 60 minutes was preferable to 15 or 30 minutes. There have been two additional studies examining optimal PIEB settings. Epsztein Kanczuk et al. [47] found an optimal PIEB time interval (effective interval 90\%) of approximately 40 minutes using $10 \mathrm{ml}$ PIEB boluses of $0.0625 \%$ bupivacaine with $2 \mu \mathrm{g} /$ $\mathrm{ml}$ of fentanyl. A subsequent study by the same group determined the ED90 optimal PIEB bolus volume when using this time interval of 40 minutes was approximately $11 \mathrm{ml}$, and that reducing bolus volume below $10 \mathrm{ml}$ resulted in inferior analgesia [48]. The rate of injection of the PIEB boluses may also improve PIEB efficacy by recruiting all the orifices in a multiport epidural catheter and generating higher injection pressures and therefore spread in the epidural space. However a recent randomized trial was unable to show any differences in labor analgesia quality or need for supplemental epidural analgesia when PIEB and PCEA boluses delivered at $100 \mathrm{ml} /$ $\mathrm{h}$ were compared to those given at $300 \mathrm{ml} / \mathrm{h}$ [49]. Higher rates of injection may also increase upstream alarms, and require specialized high-flow tubing [46].

\section{Optimal epidural solution}

Historically, epidurals consisted of concentrated local anesthetics ( $0.25 \%-0.5 \%$ bupivacaine) to achieve pain relief during labor, however these high concentrations were associated with an unacceptably high incidence of motor block

Table 1. Suggested Doses and Settings for PIEB with PCEA

\begin{tabular}{|c|c|c|}
\hline Dose/Settings & Epidural & CSE \\
\hline \multicolumn{3}{|l|}{ Initiation } \\
\hline Volume & $15 \mathrm{ml}$ & $1.1 \mathrm{ml}$ \\
\hline Solution & $\begin{array}{l}\text { 0.125\% bupivacaine } \\
+10 \mu g \text { sufentanil }\end{array}$ & $\begin{array}{l}\text { 0.25\% bupivacaine + } \\
5 \mu \text { g sufentanil }\end{array}$ \\
\hline \multicolumn{3}{|l|}{ Maintenance } \\
\hline Solution & \multicolumn{2}{|c|}{$\begin{array}{c}0.0625 \% \text { bupivacaine }+ \\
0.4 \mu \mathrm{g} / \mathrm{ml} \text { sufentanil }\end{array}$} \\
\hline PIEB bolus volume & \multicolumn{2}{|c|}{$9 \mathrm{ml}$} \\
\hline PIEB interval & \multicolumn{2}{|c|}{$\begin{array}{c}45 \text { minutes (1st bolus } 30 \text { minutes } \\
\text { after initiation) }\end{array}$} \\
\hline PCEA bolus volume & \multicolumn{2}{|c|}{$10 \mathrm{ml}$} \\
\hline PCEA lockout interval & \multicolumn{2}{|c|}{10 minutes } \\
\hline
\end{tabular}

PIEB: programmed intermittent epidural bolus, PCEA: patient-controlled epidural analgesia, CSE: combined spinal-epidural. These suggested PIEB + PCEA settings are currently utilized at Lucile Packard Children's Hospital, Stanford University, CA, USA. 
and impacted women's ability to push during the second stage of labor. The use of dilute local anesthetic solutions (i.e. $\leq 0.1 \%$ bupivacaine) is essential to minimize the impact of epidural labor analgesia on maternal mobility, duration of labor, or need for assisted vaginal delivery. Concerns about inadequate maternal analgesia with dilute local anesthetic solutions are unfounded, with excellent analgesia and high maternal satisfaction reported with dilute compared to more concentrated local anesthetic epidural solutions [50]. Chestnut et al. [51] demonstrated that there was no difference in the percentage of women reporting their labor analgesia as excellent or good when comparing $0.0625 \%$ bupivacaine +2 $\mu \mathrm{g} / \mathrm{ml}$ fentanyl to $0.125 \%$ bupivacaine alone, but that women in the higher concentration group had significantly more motor block at full cervical dilatation. A meta-analysis of randomized trials comparing low concentration labor epidural ( $<0.1 \%$ bupivacaine or equivalent potency of ropivacaine) to those with higher concentrations demonstrated decreased risk of assisted vaginal delivery, shorter duration of second stage, less motor block, and increased ability to ambulate in the low concentration group with no appreciable differences in analgesia [50]. In another meta-analysis, no differences in assisted vaginal, cesarean delivery or duration of labor were found with dilute labor epidural analgesia compared to nonepidural analgesic options [52]. Similarly, a recent large randomized study found similar duration of the second stage of labor and spontaneous vaginal delivery rates when comparing $0.08 \%$ ropivacaine with $0.4 \mu \mathrm{g} / \mathrm{ml}$ sufentanil to placebo administered with the onset of the second stage of labor [53].

The addition of a lipophilic opioid such as fentanyl or sufentanil added to local anesthetic solution reduces the minimum local anesthetic concentration required to provide adequate labor analgesia by a factor of up to 4.2 [54]. Inherent with utilizing dilute compared to concentrated local anesthetic concentrations is that larger volumes can be administered, which improves the spread within the epidural space and therefore analgesic efficacy. A minimum local anesthetic volume study by Lyons et al. [55] demonstrated equivalent analgesia at block initiation despite an overall $25 \%$ dose reduction when comparing $0.25 \%$ to $0.125 \%$ bupivacaine solution. Boselli et al. [56] found similar analgesic efficacy throughout labor despite a $30 \%$ dose reduction with $0.1 \%$ compared to $0.15 \%$ ropivacaine, both with $0.5 \mu \mathrm{g} / \mathrm{ml}$ of sufentanil.
Epidural labor analgesia that minimizes maternal side effects requires dilute local anesthetic solutions to be utilized. To facilitate the use of dilute local anesthetics while maintaining similar of levels analgesia, a lipophilic opioid must be added into the labor epidural solution. Studies comparing fentanyl and sufentanil show similar analgesic efficacy and side effects with comparative equipotent doses, so either of these opioids are appropriate. Recommended dosages of opioids are fentanyl $2-3 \mu \mathrm{g} / \mathrm{ml}$ or sufentanil $0.2-0.4 \mu \mathrm{g} / \mathrm{ml}$, although the optimal concentration depends on the concentration of local anesthetic utilized and the maintenance regimen chosen (PIEB vs. CEI +/- PCEA).

\section{CONCLUSION}

In conclusion, there are many strategies to optimize initiation and maintenance of neuraxial labor analgesia. The use of techniques such as CSE or DPE may offer benefits over standard epidurals, and the use of lumbar neuraxial ultrasound can aid in placement of any of the above-mentioned blocks and influence ultimate success of the neuraxial technique. PCEA with a background of PIEB represents the maintenance technique of choice, but more work is still required to determine the optimum settings. The use of dilute local anesthetic solutions combined with lipophilic opioids is critical to minimize the impact of labor epidural analgesia on obstetric outcomes.

Modern neuraxial techniques utilizing dilute local anesthetic solutions with PIEB + PCEA provide highly effective labor analgesia with minimal side effects. Adoption of stateof-the-art techniques outlined in this review can improve initiation and maintenance of neuraxial labor analgesia and increase maternal requests for neuraxial analgesia during labor.

\section{REFERENCES}

1. Traynor AJ, Aragon M, Ghosh D, Choi RS, Dingmann C, Vu Tran $\mathrm{Z}$, et al. Obstetric anesthesia workforce survey: a 30-year update. Anesth Analg 2016; 122: 1939-46.

2. Simmons SW, Taghizadeh N, Dennis AT, Hughes D, Cyna AM. Combined spinal-epidural versus epidural analgesia in labour. Cochrane Database Syst Rev 2012; 10: CD003401.

3. Gambling D, Berkowitz J, Farrell TR, Pue A, Shay D. A randomized controlled comparison of epidural analgesia and combined 
spinal-epidural analgesia in a private practice setting: pain scores during first and second stages of labor and at delivery. Anesth Analg 2013; 116: 636-43.

4. Heesen M, Van de Velde M, Klöhr S, Lehberger J, Rossaint R, Straube S. Meta-analysis of the success of block following combined spinal-epidural vs epidural analgesia during labour. Anaesthesia 2014; 69: 64-71.

5. Booth JM, Pan JC, Ross VH, Russell GB, Harris LC, Pan PH. Combined spinal epidural technique for labor analgesia does not delay recognition of epidural catheter failures: a single-center retrospective cohort survival analysis. Anesthesiology 2016; 125: 516-24.

6. Collis RE, Davies DW, Aveling W. Randomised comparison of combined spinal-epidural and standard epidural analgesia in labour. Lancet 1995; 345: 1413-6.

7. Grondin LS, Nelson K, Ross V, Aponte O, Lee S, Pan PH. Success of spinal and epidural labor analgesia: comparison of loss of resistance technique using air versus saline in combined spinalepidural labor analgesia technique. Anesthesiology 2009; 111: 165-72.

8. Lee S, Lew E, Lim Y, Sia AT. Failure of augmentation of labor epidural analgesia for intrapartum cesarean delivery: a retrospective review. Anesth Analg 2009; 108: 252-4.

9. Hattler J, Klimek M, Rossaint R, Heesen M. The effect of combined spinal-epidural versus epidural analgesia in laboring women on nonreassuring fetal heart rate tracings: systematic review and meta-analysis. Anesth Analg 2016; 123: 955-64.

10. Segal S, Csavoy AN, Datta S. The tocolytic effect of catecholamines in the gravid rat uterus. Anesth Analg 1998; 87: 864-9.

11. Segal S, Wang SY. The effect of maternal catecholamines on the caliber of gravid uterine microvessels. Anesth Analg 2008; 106: 888-92.

12. Abrão KC, Francisco RP, Miyadahira S, Cicarelli DD, Zugaib M. Elevation of uterine basal tone and fetal heart rate abnormalities after labor analgesia: a randomized controlled trial. Obstet Gynecol 2009; 113: 41-7.

13. Van de Velde M, Teunkens A, Hanssens M, Vandermeersch E, Verhaeghe J. Intrathecal sufentanil and fetal heart rate abnormalities: a double-blind, double placebo-controlled trial comparing two forms of combined spinal epidural analgesia with epidural analgesia in labor. Anesth Analg 2004; 98: 1153-9.

14. Pan PH, Bogard TD, Owen MD. Incidence and characteristics of failures in obstetric neuraxial analgesia and anesthesia: a retrospective analysis of 19,259 deliveries. Int J Obstet Anesth 2004; 13: 227-33.

15. Norris MC. Are combined spinal-epidural catheters reliable? Int J Obstet Anesth 2000; 9: 3-6.
16. Cappiello E, O'Rourke N, Segal S, Tsen LC. A randomized trial of dural puncture epidural technique compared with the standard epidural technique for labor analgesia. Anesth Analg 2008; 107: 1646-51.

17. Chau A, Bibbo C, Huang CC, Elterman KG, Cappiello EC, Robinson JN, et al. Dural puncture epidural technique improves labor analgesia quality with fewer side effects compared with epidural and combined spinal epidural techniques: a randomized clinical trial. Anesth Analg 2017; 124: 560-9.

18. Bernards CM, Kopacz DJ, Michel MZ. Effect of needle puncture on morphine and lidocaine flux through the spinal meninges of the monkey in vitro. Implications for combined spinal-epidural anesthesia. Anesthesiology 1994; 80: 853-8.

19. Thomas JA, Pan PH, Harris LC, Owen MD, D’Angelo R. Dural puncture with a 27-gauge Whitacre needle as part of a combined spinal-epidural technique does not improve labor epidural catheter function. Anesthesiology 2005; 103: 1046-51.

20. Weiniger CF, Sharoni L. The use of ultrasound in obstetric anesthesia. Curr Opin Anaesthesiol 2017; 30: 306-12.

21. Perlas A, Chaparro LE, Chin KJ. Lumbar neuraxial ultrasound for spinal and epidural anesthesia: a systematic review and metaanalysis. Reg Anesth Pain Med 2016; 41: 251-60.

22. Shaikh F, Brzezinski J, Alexander S, Arzola C, Carvalho JC, Beyene J, et al. Ultrasound imaging for lumbar punctures and epidural catheterisations: systematic review and meta-analysis. BMJ 2013; 346: f1720.

23. Grau T, Bartusseck E, Conradi R, Martin E, Motsch J. Ultrasound imaging improves learning curves in obstetric epidural anesthesia: a preliminary study. Can J Anaesth 2003; 50: 1047-50.

24. Vallejo MC, Phelps AL, Singh S, Orebaugh SL, Sah N. Ultrasound decreases the failed labor epidural rate in resident trainees. Int J Obstet Anesth 2010; 19: 373-8.

25. Ansari T, Yousef A, El Gamassy A, Fayez M. Ultrasound-guided spinal anaesthesia in obstetrics: is there an advantage over the landmark technique in patients with easily palpable spines? Int J Obstet Anesth 2014; 23: 213-6.

26. Grau T, Leipold RW, Conradi R, Martin E, Motsch J. Efficacy of ultrasound imaging in obstetric epidural anesthesia. J Clin Anesth 2002; 14: 169-75.

27. Moore A, Villeneuve V, Bravim B, El-Bahrawy A, El-Mouallem E, Kaufman I, et al. The labor analgesia requirements in nulliparous women randomized to epidural catheter placement in a high or low intervertebral space. Anesth Analg 2017; 125: 196974.

28. Margarido CB, Mikhael R, Arzola C, Balki M, Carvalho JC. The intercristal line determined by palpation is not a reliable anatomical landmark for neuraxial anesthesia. Can J Anaesth 2011; 
58: 262-6.

29. Balki M, Lee Y, Halpern S, Carvalho JC. Ultrasound imaging of the lumbar spine in the transverse plane: the correlation between estimated and actual depth to the epidural space in obese parturients. Anesth Analg 2009; 108: 1876-81.

30. Chin KJ, Perlas A, Chan V, Brown-Shreves D, Koshkin A, Vaishnav V. Ultrasound imaging facilitates spinal anesthesia in adults with difficult surface anatomic landmarks. Anesthesiology 2011; 115: 94-101.

31. Gambling DR, Yu P, Cole C, McMorland GH, Palmer L. A comparative study of patient controlled epidural analgesia (PCEA) and continuous infusion epidural analgesia (CIEA) during labour. Can J Anaesth 1988; 35: 249-54.

32. Van der Vyver M, Halpern S, Joseph G. Patient-controlled epidural analgesia versus continuous infusion for labour analgesia: a meta-analysis. Br J Anaesth 2002; 89: 459-65.

33. Carvalho B, Wang P, Cohen SE. A survey of labor patient-controlled epidural anesthesia practice in California hospitals. Int J Obstet Anesth 2006; 15: 217-22.

34. Halpern SH, Carvalho B. Patient-controlled epidural analgesia for labor. Anesth Analg 2009; 108: 921-8.

35. Chua SM, Sia AT. Automated intermittent epidural boluses improve analgesia induced by intrathecal fentanyl during labour. Can J Anaesth 2004; 51: 581-5.

36. Hogan Q. Distribution of solution in the epidural space: examination by cryomicrotome section. Reg Anesth Pain Med 2002; 27: 150-6.

37. Kaynar AM, Shankar KB. Epidural infusion: continuous or bolus? Anesth Analg 1999; 89: 534.

38. Mowat I, Tang R, Vaghadia H, Krebs C, Henderson WR, Sawka A. Epidural distribution of dye administered via an epidural catheter in a porcine model. Br J Anaesth 2016; 116: 277-81.

39. Wong CA, Ratliff JT, Sullivan JT, Scavone BM, Toledo P, McCarthy RJ. A randomized comparison of programmed intermittent epidural bolus with continuous epidural infusion for labor analgesia. Anesth Analg 2006; 102: 904-9.

40. Capogna G, Celleno D, Sebastiani M, Muratori F, Costantino P, Cipriani G, et al. Propofol and thiopentone for caesarean section revisited: maternal effects and neonatal outcome. Int J Obstet Anesth 1991; 1: 19-23.

41. George RB, Allen TK, Habib AS. Intermittent epidural bolus compared with continuous epidural infusions for labor analgesia: a systematic review and meta-analysis. Anesth Analg 2013; 116: $133-44$

42. Sng BL, Zeng Y, de Souza NNA, Leong WL, Oh TT, Siddiqui FJ, et al. Automated mandatory bolus versus basal infusion for maintenance of epidural analgesia in labour. Cochrane Database Syst
Rev 2018; 5: CD011344.

43. McKenzie CP, Cobb B, Riley ET, Carvalho B. Programmed intermittent epidural boluses for maintenance of labor analgesia: an impact study. Int J Obstet Anesth 2016; 26: 32-8.

44. Tien M, Allen TK, Mauritz A, Habib AS. A retrospective comparison of programmed intermittent epidural bolus with continuous epidural infusion for maintenance of labor analgesia. Curr Med Res Opin 2016; 32: 1435-40.

45. Delgado C, Ciliberto C, Bollag L, Sedensky M, Landau R. Continuous epidural infusion versus programmed intermittent epidural bolus for labor analgesia: optimal configuration of parameters to reduce physician-administered top-ups. Curr Med Res Opin 2018; 34: 649-56.

46. Carvalho B, George RB, Cobb B, McKenzie C, Riley ET. Implementation of programmed intermittent epidural bolus for the maintenance of labor analgesia. Anesth Analg 2016; 123: 965-71.

47. Epsztein Kanczuk M, Barrett NM, Arzola C, Downey K, Ye XY, Carvalho JC. Programmed intermittent epidural bolus for labor analgesia during first stage of labor: a biased-coin up-and-down sequential allocation trial to determine the optimum interval time between boluses of a fixed volume of $10 \mathrm{~mL}$ of bupivacaine 0.0625\% with fentanyl $2 \mu \mathrm{g} / \mathrm{mL}$. Anesth Analg 2017; 124: 537-41.

48. Zakus P, Arzola C, Bittencourt R, Downey K, Ye XY, Carvalho JC. Determination of the optimal programmed intermittent epidural bolus volume of bupivacaine $0.0625 \%$ with fentanyl $2 \mu$ g.ml-1 at a fixed interval of forty minutes: a biased coin up-and-down sequential allocation trial. Anaesthesia 2018; 73: 459-65.

49. Lange EMS, Wong CA, Fitzgerald PC, Davila WF, Rao S, McCarthy RJ, et al. Effect of epidural infusion bolus delivery rate on the duration of labor analgesia: a randomized clinical trial. Anesthesiology 2018; 128: 745-53.

50. Sultan P, Murphy C, Halpern S, Carvalho B. The effect of low concentrations versus high concentrations of local anesthetics for labour analgesia on obstetric and anesthetic outcomes: a meta-analysis. Can J Anaesth 2013; 60: 840-54.

51. Chestnut DH, Owen CL, Bates JN, Ostman LG, Choi WW, Geiger MW. Continuous infusion epidural analgesia during labor: a randomized, double-blind comparison of $0.0625 \%$ bupivacaine $/ 0.0002 \%$ fentanyl versus $0.125 \%$ bupivacaine. Anesthesiology 1988; 68: 754-9.

52. Wang TT, Sun S, Huang SQ. Effects of Epidural labor analgesia with low concentrations of local anesthetics on obstetric outcomes: a systematic review and meta-analysis of randomized controlled trials. Anesth Analg 2017; 124: 1571-80.

53. Shen X, Li Y, Xu S, Wang N, Fan S, Qin X, et al. Epidural analgesia during the second stage of labor: a randomized controlled trial. Obstet Gynecol 2017; 130: 1097-103. 
54. Buyse I, Stockman W, Columb M, Vandermeersch E, Van de Velde M. Effect of sufentanil on minimum local analgesic concentrations of epidural bupivacaine, ropivacaine and levobupivacaine in nullipara in early labour. Int J Obstet Anesth 2007; 16: 22-8.

55. Lyons GR, Kocarev MG, Wilson RC, Columb MO. A comparison of minimum local anesthetic volumes and doses of epidural bupivacaine $(0.125 \% \mathrm{w} / \mathrm{v}$ and $0.25 \% \mathrm{w} / \mathrm{v})$ for analgesia in labor. Anesth Analg 2007; 104: 412-5.

56. Boselli E, Debon R, Duflo F, Bryssine B, Allaouchiche B, Chassard D. Ropivacaine $0.15 \%$ plus sufentanil $0.5 \mathrm{microg} / \mathrm{mL}$ and ropivacaine $0.10 \%$ plus sufentanil $0.5 \mathrm{microg} / \mathrm{mL}$ are equivalent for patient-controlled epidural analgesia during labor. Anesth Analg 2003; 96: 1173-7. 